\title{
System for Edge Detection and Following Boundary in Medical Images (SEB)
}

\author{
Sahera Abued Sead \\ University of Basrah \\ College of Computer Science and Information \\ Technology, \\ Basrah, Iraq
}

\author{
Hawraa Sabah Naser \\ University of Basrah \\ College of Science \\ Basrah, Iraq
}

\begin{abstract}
The use of medical images has increased dramatically, including medical surveillance and diagnosis, and there is a growing need for accurate data access. Edge and boundary detection plays an essential role in the analysis and interpretation of image contents and is one of the goals in computer vision. The object of the boundary detection basically is to find the objects in the images i.e. the boundaries of the objects in the image are located. Detecting the right boundary in the noisy images is still a very difficult task. There were several ways to resolve this issue, such as ACM models, but it is still difficult to process images in concave boundaries and noisy areas. A new system for detecting edges and boundary following in noisy images has been introduced. It has been applied to a variety of medical MRI images.
\end{abstract}

The proposed system consists of two models: the edge detection model and the boundary following model. Prior to each pre-processing model, a set of processing were included for the purpose of image preparation the main purpose of this pre-processing is to use the discrete curvelet transform to enhance the images, much more efficient than traditional transformations to improves the vision and deletes noise. The first model in the proposed system detects the edges of the objects in the image, depending on the texture feature image and Edge Mapping.

The second model of the system is in the process of following the boundaries, The following process was based on the :first, finding the average vector ,the most important features of this process eliminates random directions will give better results and more accurate and increases the clarity of the edges in the image. Second, find the starting point of the boundary using the idea of the density of the edge length and calculate the values of the connection to each point and then starting the moving algorithm work from the starting point of the boundary and move on the boundary points by choosing the appropriate point of the transition points.

The efficiency and effectiveness of the proposed system can be analyzed through the results of experiments that showed that the performance of the system is very good and gives excellent and more accurate results, by comparing the results of the detection model with the traditional methods of edge detection such as sobel, prewitt and canny and compare the following model with ACM models. The results showed that the proposed system gives better and faster performance than these models. The running time of the proposed system, the edge detection model took about (13.9020) seconds. The following model took (0.3649) seconds and the total running time of the system was approximately (19.3919) seconds.

\section{General Terms}

Edge Detection, Boundary Following

\author{
Keywords \\ Texture Segmentation, Curvelet Transform, Vector, Start \\ Point, Law's Mask
}

\section{INTRODUCTION}

Living in the era of information technology therefore, most of the techniques require digital image and process it in the digital domain. Especially the medical image because its usage is increasing tremendously in different medical situations therefore, it became an obvious interest to develop and create more efficient and fast methods to carry out this processing[1][2][3]. Edge detection from computer vision applications and the other applications is considered very important initial step which considers the core of the process want to do such as study, analysis. In medical space find the correct edge or boundary of the object which considers a very critical process because it gives useful information which helps in diagnosis of tumors, tissues and others. Because of the complex nature of the medical images and the noise, they get because of the way the image is captured, the device used, the intensity of light and others, cause noise affecting the edge and making it difficult to correctly detect the edge in a lot of images[4][5][6][7]. Where the edge detection is used to detect the different edges in the image such as Canny, Sobel and Laplacian etc., The Edge detection methods depend in basic on the difference of the gray levels in the image which used to detect the discontinuity. The disadvantage of Sobel edge detection are sensitivity to noise, inaccurate and with coarse edge width its detect the false edge.

laplacian edge detection disadvantage are responding to some of the existing edges, sensitivity to noise, magnitude the Laplacian produce double edge and its unable to detect the direction of the edge. Canny disadvantages are timeconsuming and complex computations [8][9]. These methods share a common problem in that they do not detect the details of edges, and as they fail to extract the correct boundaries of objects in noisy images. There are new methods which used to solve the problem detect and following the boundary of the object in an image known as Active Contour Model (ACM) however, the snake has weaknesses and limitations of small capture range and difficulties progressing into concave boundary regions, also it's sensitive to the noise and small objects.

Most of the methods of snake models require a very accurate initial contour estimate of the object such as detect several points around the object by using the mouse or enter the points manually which consider hard problem especially for people unfamiliar with the subject. The contour of snake models can converge to a wrong boundary if the initial contour is not close enough to the desired boundary[3][10][11][12]. This technique also requires proper initialization of the initial contour, which is not too far from the expected boundary. ACM is also difficult to implement in 
real-time and often require careful tuning of parameters for convergence. After this simplified illustration of the importance of detecting and following boundary especially in medical images and deficiencies in traditional methods came the need to design a system that can detect edges and following boundaries accurately and efficiently.

In this paper is organized as follows. Section 2 describes the details of the proposed system. The experimental results of the proposed in section 3 .Finally, section 4 presents the conclusions for this work.

\section{THE PROPOSED SYSTEM}

The proposed system consist of two different models shares the preprocess stage, and will be clarified in follow:

\subsection{Pre-Processing Stage}

This process is intended to create images for processing, including sequential steps, after image processing. Image processing processes (this process is performed for the purpose of obtaining images of the appropriate size for handling the storage and time), The process of converting the image to gray, and the image enhancement process for noise management from image capture where the process is done using Fast Discrete Curvelet Transform via Wrapping (FDCT), because of the FDCT efficiency in represents images at various scales and different angles and this was designed originally to represent edges and curves well than wavelet and this transform uses rapid fourier transform which has fewer computational complexities and to preserve the image features along certain directions.

This operation trying to make the image more visible appearance especially the medical image to become more suitable and useful for analysis, detect the edge or feature extraction. Curvelet was suitable to processing the wave propagation data because of its ability to obtain full spectral information of an image with different directions of image edges. Curvelet transform is considered a good filter to enhance and denoising the image. figure (1) illustrates the application of the enhancement process on some images.

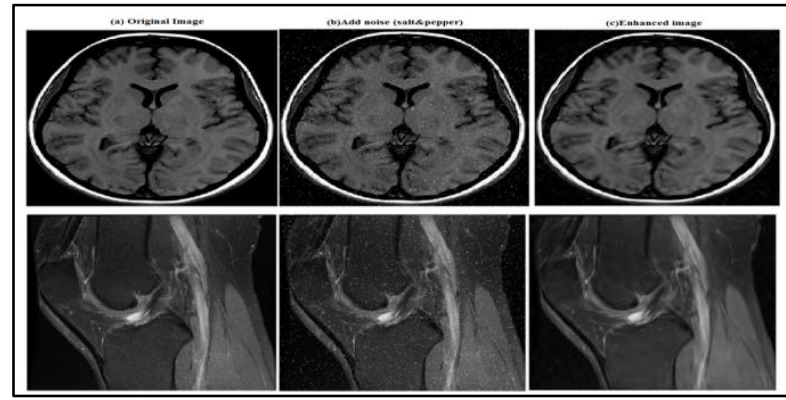

Fig 1: Apply of the enhancement process (a) Original images (b) Images after adding noise (c) Enhanced images

\subsection{EDGE DETECTION MODEL}

The edges are detection in this model for the input images by finding edge mapping and depending on the texture feature image. Edge detection model consists from the following operations:

\subsubsection{Create 2-D Law's Filters}

In this process law's texture masks are initialized which will use for filtering, this masks is one-dimension vector as following:-

$$
\begin{aligned}
& \text { L5 (level) }=\left[\begin{array}{lllll}
1 & 4 & 6 & 4 & 1
\end{array}\right] \\
& \text { E5 (Edge) }=\left[\begin{array}{lllll}
-1 & -2 & 0 & 2 & 1
\end{array}\right]
\end{aligned}
$$

$$
\begin{aligned}
& \text { R5 (Ripple) }=\left[\begin{array}{lllll}
1 & -4 & 6 & -4 & 1
\end{array}\right] \\
& \text { S5 (Spot) }=\left[\begin{array}{lllll}
-1 & 0 & 2 & 0 & -1
\end{array}\right]
\end{aligned}
$$

The first step before using the these vectors is converting the one-dimension vector of law's texture to two-dimension masks by multiplying each mask from one-dimensional masks with all masks, for example if the two E5 , L5 masks are taken the two-dimension mask is compute as:-

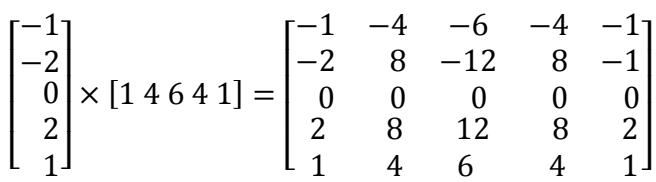

\subsubsection{Convolution between Image and Law's}

\section{Filters}

In this process, the input image is filtered the texture filters of the law's texture, which calculated by convolution the input image with each of the sixteen law's masks, as shown in figure(2)and this is done by equation (1).

$\hat{f}_{k}(i, j)=f(i, j) * h_{k}$

Where $h_{k}$ represent the micro-texture filters.

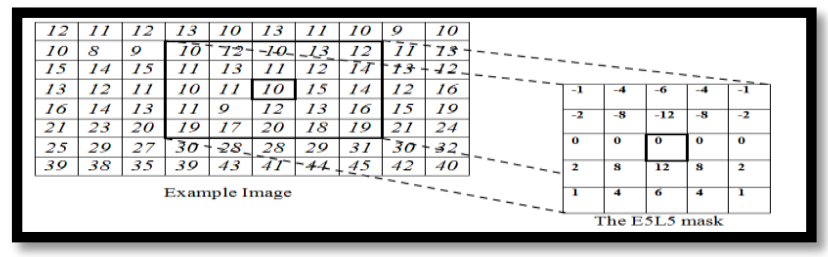

Fig 2: Convolution between image and E5L5 mask

The nine features are then determined by replacing each pair with its mean and as follows: [L5E5 IE5L5, L5S5\S5L5,

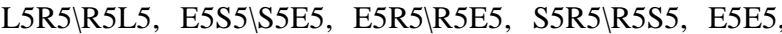
S5S5, R5R5]. The nine remaining masks are: [ L5E5, L5R5, E5S5, S5S5 ,R5R5 , L5S5, E5E5 , E5R5, S5R5] Therefore, the output of this process will be nine images, each image represents one of the characteristics of the texture of the image as shown in figure (3).
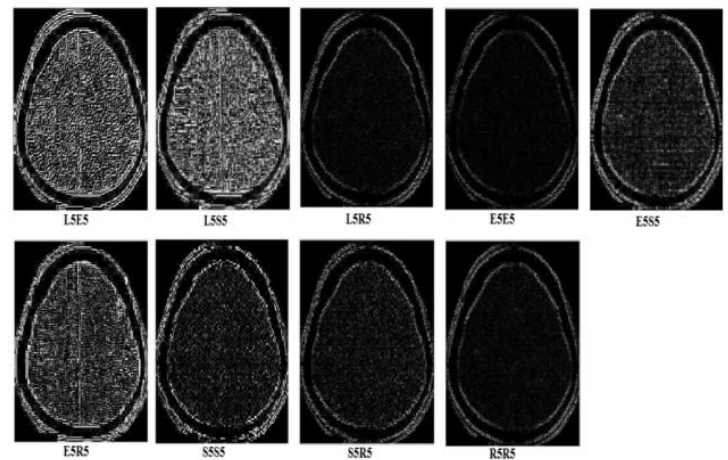

Fig 3: law's texture Masks Application on the Image

\subsubsection{Summation of Texture Energy}

Calculate the energy of the texture by collecting the absolute value of the filter output of the local neighbors around a point in order to obtain the nine characteristics as equation(2). As shown in figure (4), then the characteristics (feature) are additional as shown in figure (5).

$$
\hat{f}_{k}(i, j)=\frac{1}{w^{2}} \sum_{n=-w / 2}^{w / 2} \sum_{m=-w / 2}^{w / 2}\left|\hat{f}_{k}(n, m)\right|
$$


Where $\mathrm{w}$ is size of the macro-window.

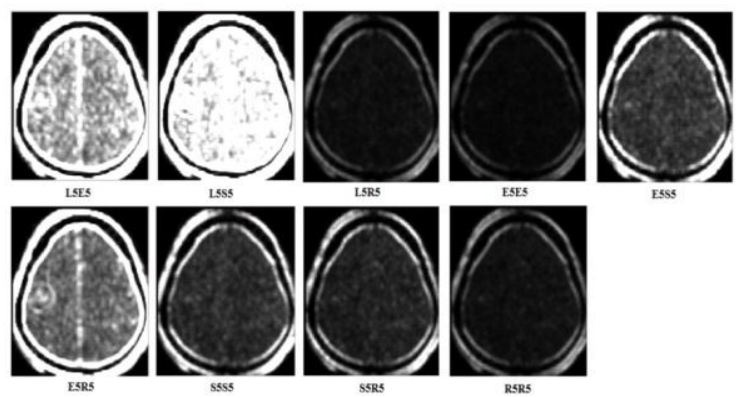

Fig 4: Law's Energy Texture Image

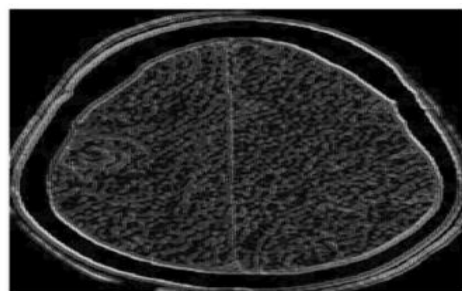

Fig 5: Law's Final Image

\subsubsection{Find Edge Mapping}

This process take the result of the previous process and put it as input to canny edge detection, make convolution to the image which produced by law's texture with gaussian masks, then compute magnitude and direction of the gradient, then remove non-maximum values to detect the edges, then select the threshold as shown in figure(6) and figure(7).

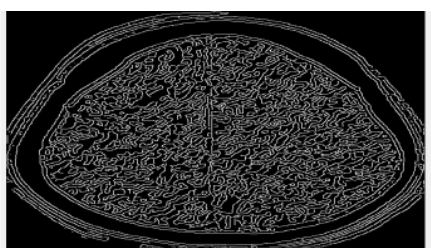

Fig 6: Edge Mapping Model
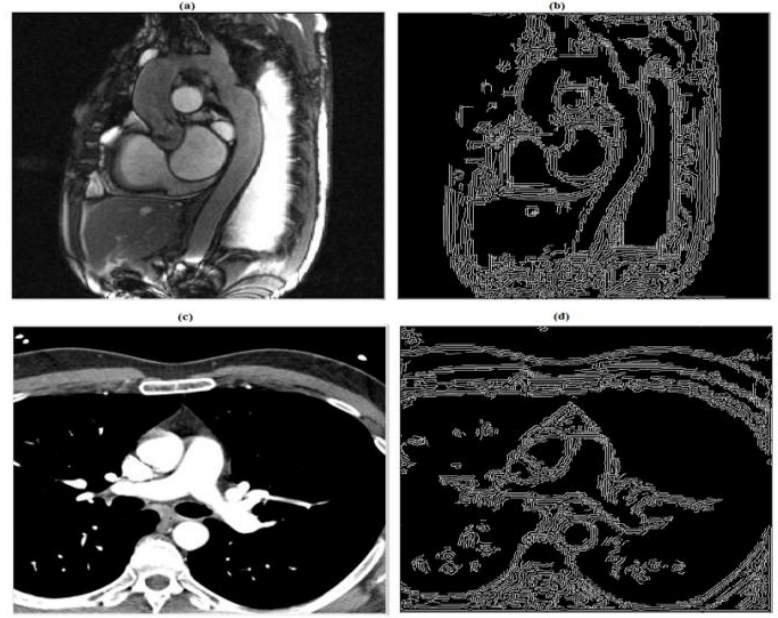

Figure 7: (A) Image of the heart (b)Edge map of the hear (c)Image of the lung (d) Edge map of the lung

\subsection{Boundary Following Model}

In this model the boundary of the object in the image is detect where in the first step the vector image is calculated which detect the magnitude and direction of the edges in the image, the boundary following model consist of the following steps:-

\subsubsection{Convolution between Image and Gaussian Masks}

In this operation each pixel in the image convolved with gaussian mask by using gaussian operator, and this operators are the directions $x$ and y respectively. The equations (3) and (4) explain how this step applied.

$$
\begin{aligned}
& C O_{x}(i, j)=-G M_{y} \times I(x, y) \approx \frac{\partial I(x, y)}{\partial y} \\
& C O_{y}(i, j)=G M_{x} \times I(x, y) \approx-\frac{\partial I(x, y)}{\partial x}
\end{aligned}
$$

The input image $(x, y), G M_{x}$ and $G M_{y}$ represent the gaussian weighted masks in directions $\mathrm{x}$ and $\mathrm{y}$, where the masks computed by using the following equations (5) and (6) and as shown in figure(8).
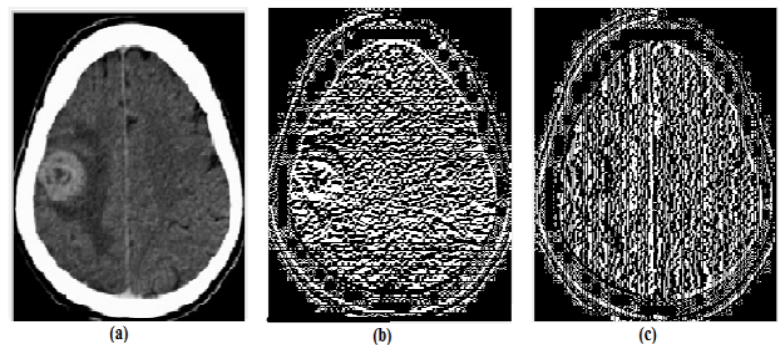

Fig 8: (a) Input image (b)Convolution in $x$-direction (c) Convolution in $y$-direction

$G M_{x}(x, y)=\frac{1}{\sqrt{2 \pi \sigma}}\left(\frac{x}{\sqrt{x^{2}+y^{2}}}\right) \exp \left(-\frac{x^{2}+y^{2}}{2 \sigma^{2}}\right)$
$G M_{y}(x, y)=\frac{1}{\sqrt{2 \pi \sigma}}\left(\frac{y}{\sqrt{x^{2}+y^{2}}}\right) \exp \left(-\frac{x^{2}+y^{2}}{2 \sigma^{2}}\right)$

\subsubsection{Calculate the Maximum Number of Points}

In this step the maximum number of the pixels in vertical and horizontal direction is computed to use it in calculation of the information of the vector and this compute using the equation (7)

$$
M P=\max _{i, j}\left(\sqrt{C O_{x}(i, j)^{2}+C O_{y}(i, j)^{2}}\right)
$$

\subsubsection{Operation Compute Vector Information}

In this step the vector information is computed by using the following equation (8):

$\operatorname{VI}(i, j)=\frac{1}{M P}\left(C O_{x}(i, j) \vec{\imath}+\operatorname{CO}_{y}(i, j) \vec{\jmath}\right)$

\subsubsection{Operation Compute Average Vector}

In unclear and noisy images the vector image is distributed randomly to all pixels in image, the portability of the vector image has been expanded by apply the local average operation, where the value of each vector is replace with the eight local average of the vector neighbors and for each pixel in image. The average vector is computed by using the equation (9).

$A V(i, j)=\frac{1}{N_{P}} \sum_{(i, j) \in N} \sqrt{C O_{x}(i, j)^{2}+C O_{y}(i, j)^{2}}$

Where N_P is the total number of the pixel and its neighbors and the direction is calculated according to equation (10). figure (9) show the different between the vector image and 
average vector.

$D(i, j)=\frac{1}{N_{p}} \sum_{(i, j) \in N} \tan ^{-1}\left(\frac{C O_{y}(i, j)}{C O_{x}(i, j)}\right)$

(a)

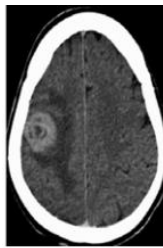

(b)

(c)

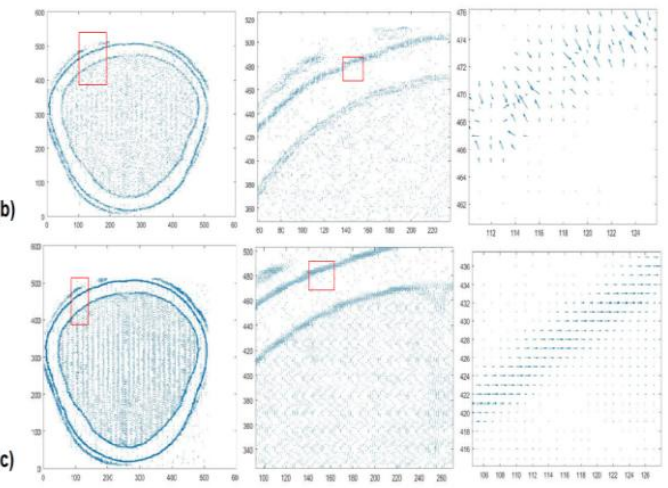

Figure (9):- (a) the input image (b) Vector Image (c) Average Vector

\subsubsection{Operation Find the Starting point of}

\section{boundary}

In this step the best point is determined for the start following the boundaries of the object, figure (10) shows the flowchart for this process.

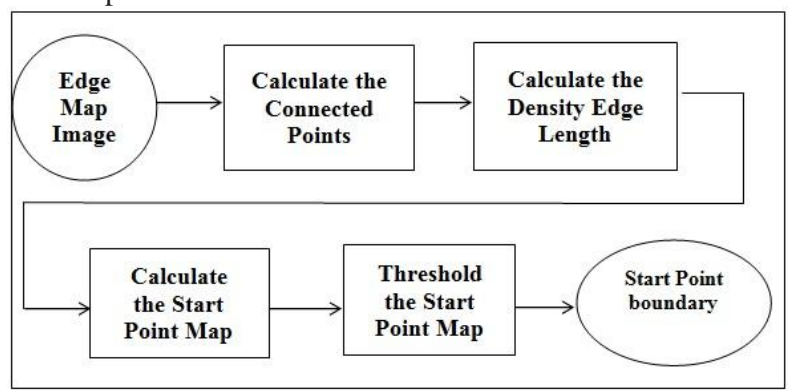

Fig 10: Finding starting point of boundary

The steps of the chart can be illustrated as follows:

\subsubsection{Compute the Connected Points}

In this step the number of the connected pixels for each pixel in the produced image of the edge map with the other point neighbors to it, where for each pixel in the image compute the connected pixels in vertical and horizontal direction, this is done for all points in the image if the pixel contain the same value of its neighbor it consider connected pixels and one will add to connect counter for each pixel, where there is two connected counter the first one for horizontal connected pixels (Num_con_hor_points) and the second for vertical direction connected pixels (Num_con_ver_points) then add the value of these counters to get the pixels connect value(Num_connect_points) and explain this step in the example in figure(11).

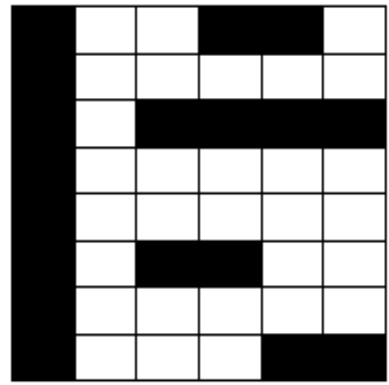

\begin{tabular}{|l|l|l|l|l|l|}
\hline 8 & & & 2 & 2 & \\
\hline 8 & & & & & \\
\hline 8 & & 4 & 4 & 4 & 4 \\
\hline 8 & & & & & \\
\hline 8 & & & & & \\
\hline 8 & & 2 & 2 & & \\
\hline 8 & & & & & \\
\hline 8 & & & & 2 & 2 \\
\hline
\end{tabular}

Fig 11: (a) The Connected Points (b) Number of Connected Points

\subsubsection{Calculate the Density Edge Length}

The idea behind using the density is to obtain the edge length, the value of density can computed for each pixel in the image by find the maximum value of the connected pixels

Max(Num_con_points) which computed in the previous step then divide each value of connected value on it as show in the equation(11)

Density_Edge_Length $(i, j)=\frac{\text { Num_con_points }(i, j)}{\operatorname{Max}(\text { Num_con_points })}(11)$

Where the Num_con_points contain the value of connected pixels for the image, applying this operation on the previous example will lead to the following result in figure (12) ,the figure (13) shows the result of applying this process to the image.

\begin{tabular}{|l|l|l|l|l|l|}
\hline 1 & & & 0.25 & 0.25 & \\
\hline 1 & & & & & \\
\hline 1 & & 0.5 & 0.5 & 0.5 & 0.5 \\
\hline 1 & & & & & \\
\hline 1 & & 0.25 & 0.25 & & \\
\hline 1 & & & & & \\
\hline 1 & & & & & \\
\hline 1 & & & & 0.25 & 0.25 \\
\hline
\end{tabular}

Fig 12 : Density of Edge Length example

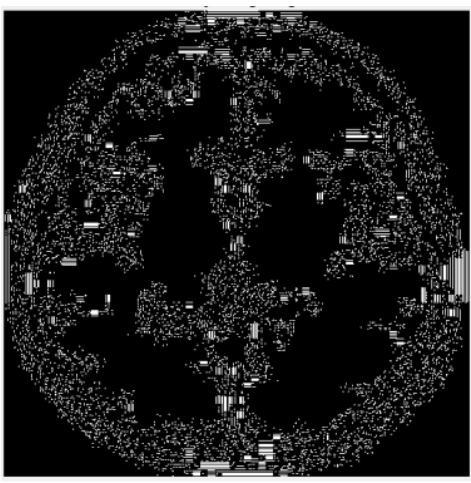

Fig 13 : Density of Edge Length

\subsubsection{Compute the Start Point Map}

The map of starting pixel in the image is computed by add the average vector image with the density of edge length as show in the following equation (12).

Ini_Pos_map $(i, j)=\frac{1}{2}(A V(i, j)+$ Density_Edge_Length $(i, j))$ 


\subsubsection{Threshold the Start Point Map}

To access the position of the starting point of the boundary, a threshold (T_Max ) must be set for the map, where the threshold is determined by equation (13)

T_Max $=\operatorname{Max}($ Ini_Pos_map $) *(90 / 100)$

Then compare each points in start point map of boundary, where if the Ini_Pos_map(i,j) $>$ T_Max then this position is good candidate to be the starting point of the boundary. Figure(14) explain the candidate points to starting of the boundary, where any point of these is a good candidate to starting point of the boundary, in the proposed system the maximum value was chosen to be the starting point.

Figure (14.c) explain the start_point_boundary which start moving to following the boundary from it and end with it, where this example its value was (72.061) and the position $(18,275)$.

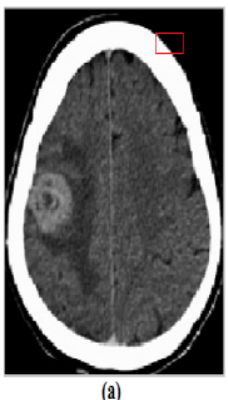

(a)

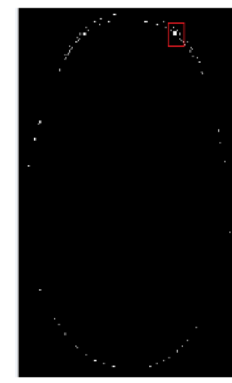

(b)

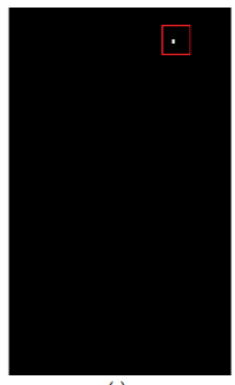

(c)
Fig 14: (a)Original Image (b)The Candidate Points (c) The Starting Point

\subsubsection{Moving on the Boundary Points}

In this operation the boundary is following from the starting point of the boundary which is detected in the previous step, in each time three pixels is candidate and start search to the more suitable pixel to move to it as explain in algorithm following:-

Algorithm( Moving on the Boundary Points):

Step1: Choose three points with largest absolute value from the average vector value from the eight neighbors around the starting point) with detecting the direction of each point of this three points with the starting point and depending on the number of the direction in figure(16).

Step2: Sort the three chosen value in descending order and store them in chosen points list.

Step3: Choose the maximum value from the three points.

Step4: Did the current point equal to the starting point or the list of the points chosen end? if yes go to end, else :

Step5: Move on step forward in the determined direction (dir) of the chosen point.

Step6: Store the current point which move to it in chosen points list which it visited.

Step7: Choose three points in direction dir,dir-1,dir+1(mod 8).
Step8: Sort the three chosen value in descending order and store them in chosen points list.

Step9: Choose the maximum value from the three maximum values.

Step10: Did the current chosen point not visited (by search it in the visited points list) if the yes move to it and delete the three value from the list and go to step 4, else:-

Step11: Did the three points in the points list not end? if yes take the next point and go to step 10, else:-

Step12: Delete the three points from the points list(move one step backward) and go to step 4 .

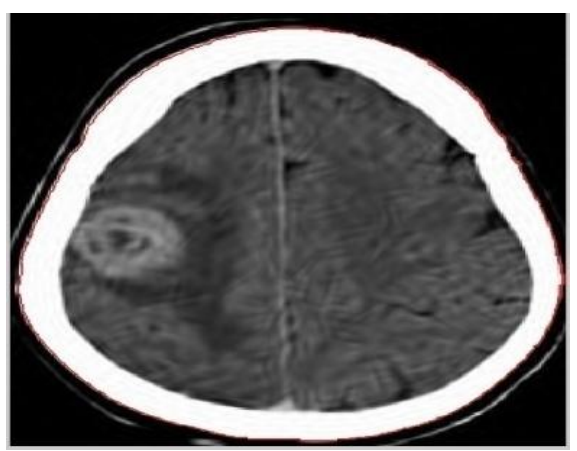

Fig 15: Boundary Following

\begin{tabular}{|c|c|c|}
\hline 0 & 1 & 2 \\
\hline 7 & $*$ & 3 \\
\hline 6 & 5 & 4 \\
\hline
\end{tabular}

Fig 16: The window of determine the direction of the each point (* the current point)

\section{RESULTS}

The proposed system has been applied and tested on various medical images type MRI images from the database ( MRI MS DB), and in different sizes where size are unified in size $(512 * 512)$ For processing. In addition, the requirements for applying the proposed system can be clarified in Table (1) The experiments and results will be explained in the follow :

Table 1. The proposed system tools

\begin{tabular}{|l|l|}
\hline \multicolumn{1}{|c|}{ Type } & The Description \\
\hline Windows Version & Windows 7 pro 64bit \\
\hline Programming language & Matlab R2016a \\
\hline Processor & Intel Core i5 \\
\hline RAM & $4.00 \mathrm{~GB}$ \\
\hline HDD & $500 \mathrm{~GB}$ \\
\hline
\end{tabular}


Experiment(1):-The proposed system was applied to a section of the kidney image as shown in figure (17).
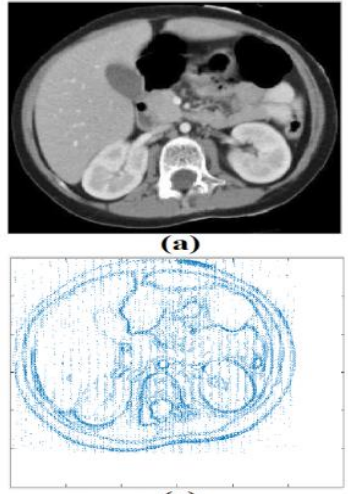

(c)

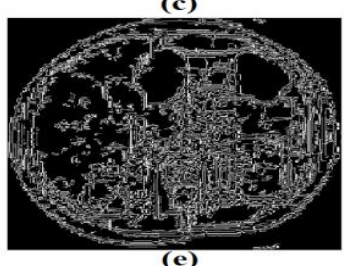

Fig 17: Experiment (1) (a)The input image (b)The enhanced image (c) Average vector image (d) Law's texture image (e) Edge map (f) Boundary following

Experiment (2): The proposed system was applied to a section of the brain image as shown in figure (18).
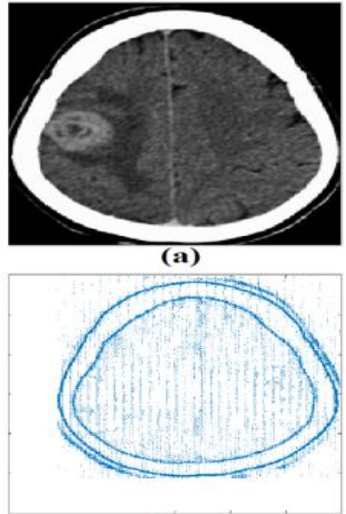

(c)

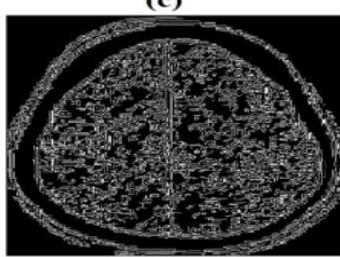

(e)
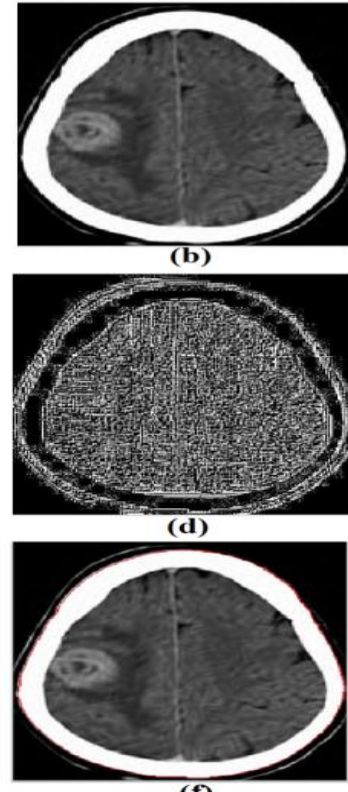

Fig 18: Experiment (2) (a)The input image (b)The enhanced image (c) Average vector image (d) Law's texture image (e) Edge map (f) Boundary following

Experiment (3): - The proposed system was applied to a section of the brain image as shown in figure (19).

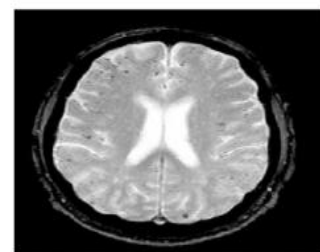

(a)

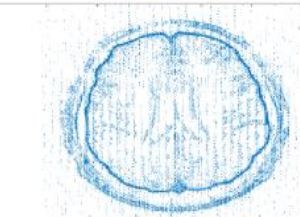

(c)

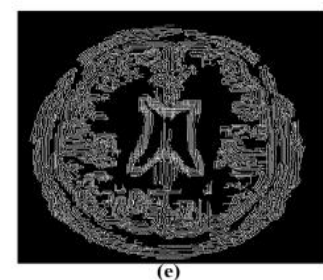

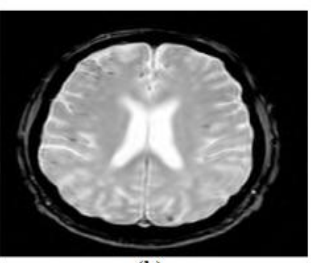

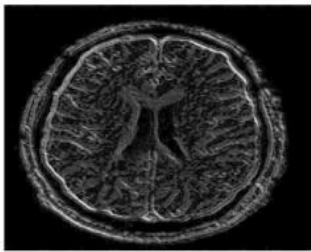

(d)

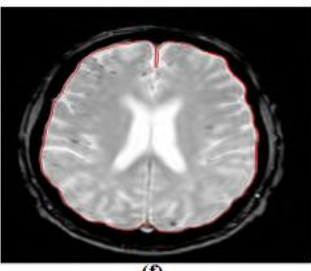

Fig 19 : Experiment (3) (a)The input image (b)The enhanced image (c) Average vector image (d) Law's texture image (e) Edge map (f) Boundary following

Experiment 4: - The proposed system was applied to a section of the kidney image as shown in figure (20).

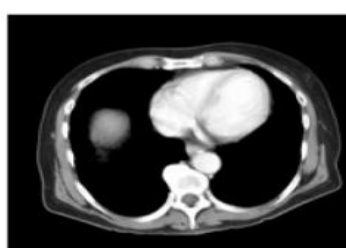

(a)

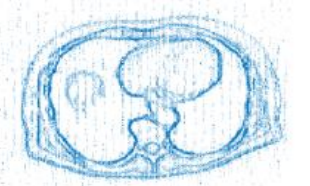

(c)

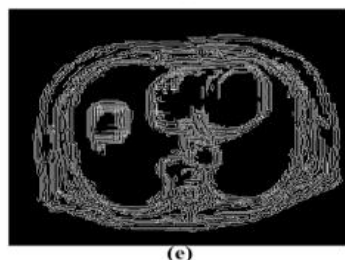

Fig 20: experiment (4) (a)The input image (b)The enhanced image (c) Average vector image (d) Law's texture image (e) Edge map (f) Boundary following

Table 2. Start_Point_Boundary Position

\begin{tabular}{|c|c|}
\hline No.Experiment & Start_Point_Boundary \\
\hline 1 & $(12,331)$ \\
\hline 2 & $(18,275)$ \\
\hline 3 & $(91,340)$ \\
\hline 4 & $(288,442)$ \\
\hline
\end{tabular}




\subsection{Comparison of the Results}

In most sciences the researchers they assess the performance of a particular technique by conducting a series of systematic scientific experiments and determining the efficiency of performance in a clear objective manner. In the case of the detection of the edges, the actual evaluation of performance is based on the human vision system, such as looking at the results.

Visual inspection of the results indicates that the detection of edges and boundary is of good quality and suitable for applications. This section presents the relative performance of various edge detection techniques such as Sobel, Prewitt, Roberts, canny and the active contour model (ACM) to verify the efficiency and accuracy of the proposed system, figures(21),(22) show the images obtained using different edge detection techniques as well as proposed system.
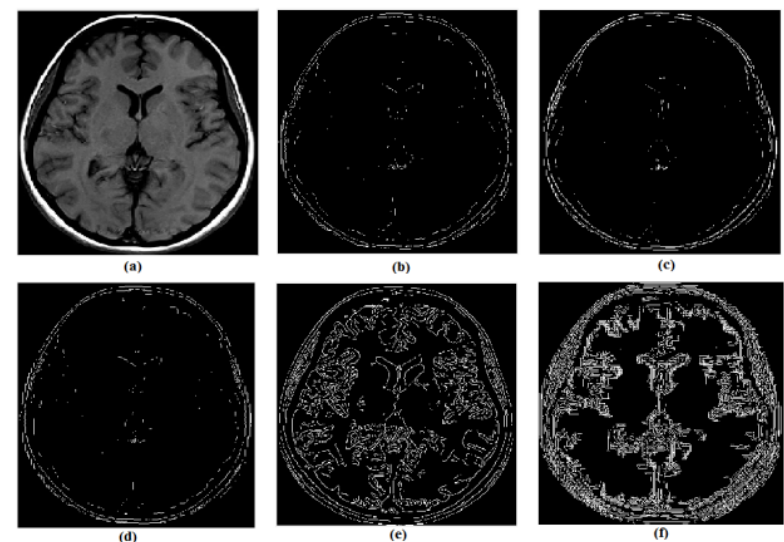

Fig 21: Edge Detection Techniques Experiment(1) (a) Original image (b) Sobel (c) Roberts (d) Prewitt (e) Canny (f) Proposed System

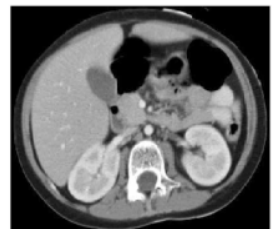

(a)

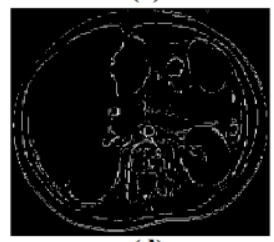

(d)

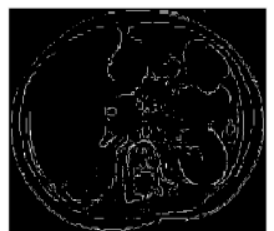

(b)

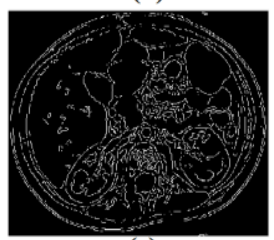

(e)

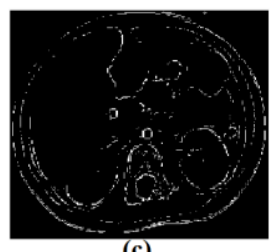

(c)

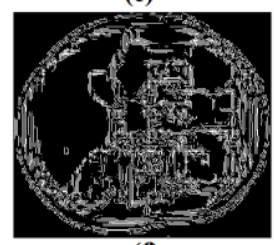

(f)
Fig 22: Edge Detection Techniques Experiment(2) (a) Original image (b) Sobel (c) Roberts (d) Prewitt (e) Canny (f) Proposed System

Note that the edges detected in the proposed system are more than the edges detected in the other methods by using the texture and the edge map. The proposed system were able to detect undetected edges in the traditional methods indicated, thus increasing the detection efficiency for use it in the applications which requiring more quantitative detection of the edge to the object. figures (23),(24),(25) Shows a comparison between the proposed boundary following and the (ACWE, ACM, Region Based Active Contours, localized Chan-Vese) to find the boundary by observation the detected boundary for these methods less efficient than the proposed system and it also time consuming (i.e. the implementation of the method (c) in figure (23) take more than seven minutes and 3000 iteration because it compute the internal energy and stop to fix the boundary by compute the external energy to estimate the boundary which is need more time and iteration.
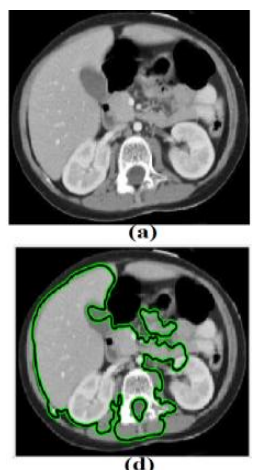

(d)
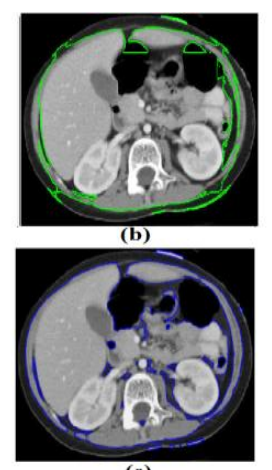
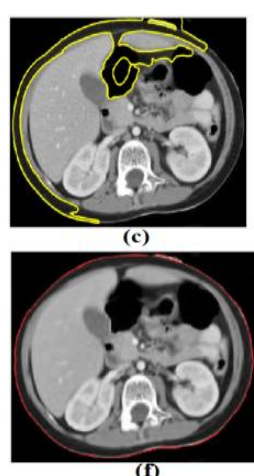

(f)
Fig 23 : Following Methods Experiment(1) (a) Original image (b) ACM (c)localized Chan-Vese (d) Region Based Active Contours (e) ACWE (f) The proposed system
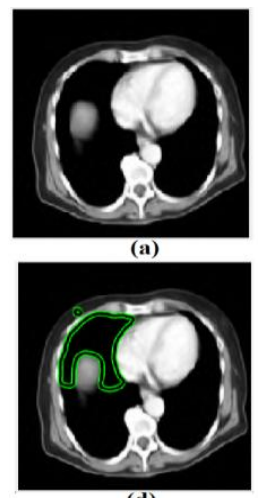

(d)
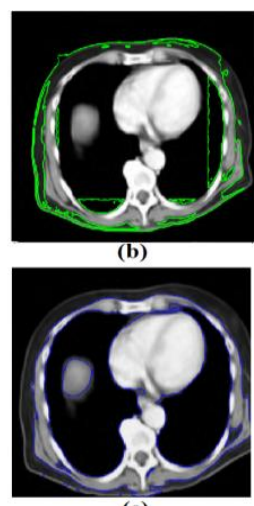

(e)
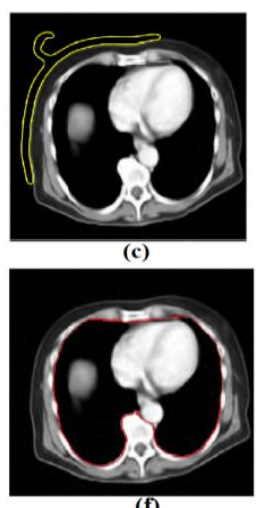

(f)
Fig 24: Following Methods Experiment(2) (a)Original image (b) ACM (c)localized Chan-Vese (d) Region Based Active Contours (e) ACWE (f) The Proposed System
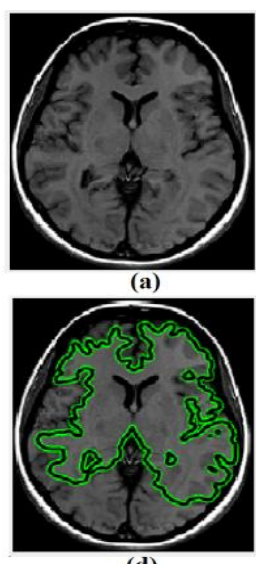

(d)
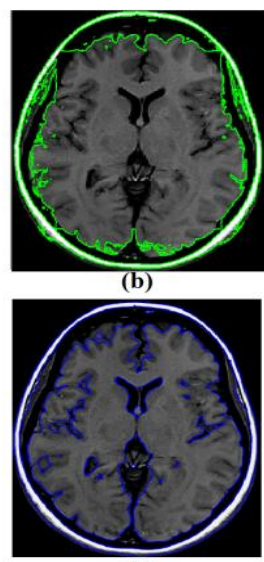
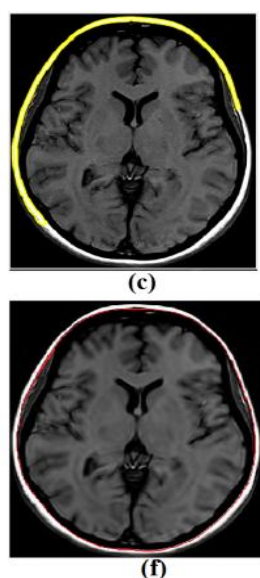

Fig 25: Following Methods Experiment(3) (a)Original image (b) ACM (c)localized Chan-Vese (d) Region Based Active Contours (e) ACWE (f) The Proposed System

The proposed system can evaluate by compute the Hausdorff distance and the mean square error (MSE) and the peak signal to noise ratio (PSNR) as shown in table(3) the result of these operations to different medical images. 
Table 3. Performance Parameters

\begin{tabular}{|c|c|c|c|}
\hline No. & Hausdorff & (MSE) & (PSNR) \\
\hline 1 & 1.3444 & 0.0065163 & 69.9908 \\
\hline 2 & 1.4634 & 0.0071735 & 69.5735 \\
\hline 3 & 1.1892 & 0.005168 & 70.9976 \\
\hline 4 & 1.7572 & 0.0080411 & 69.0776 \\
\hline 5 & 2.4306 & 0.01461 & 66.4843 \\
\hline 6 & 2.4651 & 0.013307 & 66.8901 \\
\hline
\end{tabular}

The table(3) explain that when the PSNR is increase is the best and vice versa with MSE. The Hausdorff distance can explain as a method to compute the maximum range measure between the produced image and the original image and this measure represent the similarity between these two images.

\subsection{Running Time}

We can observe the average of the time (time execution) in system stages implementation by computing the running time of the proposed system in six different image as shown in table (4) where the table explain the running time of the system stages per second for the preprocessing stage, edge detection stage, boundary following stage and law's texture compute.

Table 4. The execution time of the Proposed System stages in seconds (Per second).

\begin{tabular}{|c|c|c|c|c|}
\hline $\begin{array}{c}\text { No. } \\
\text { Experiments }\end{array}$ & $\begin{array}{c}\text { Preprocess } \\
\text { Time }\end{array}$ & $\begin{array}{c}\text { Edge } \\
\text { Detection } \\
\text { Model } \\
\text { Time }\end{array}$ & $\begin{array}{c}\text { Boundary } \\
\text { Following } \\
\text { Model } \\
\text { Time }\end{array}$ & $\begin{array}{c}\text { Law's } \\
\text { Texture } \\
\text { Time }\end{array}$ \\
\hline 1 & 5.7697 & 13.5740 & 0.3864 & 10.6211 \\
\hline 2 & 5.7822 & 13.5039 & 0.3575 & 10.6185 \\
\hline 3 & 5.8953 & 14.2967 & 0.4095 & 10.6341 \\
\hline 4 & 5.8081 & 13.6509 & 0.3782 & 10.7758 \\
\hline 5 & 4.1340 & 14.4081 & 0.3225 & 10.8033 \\
\hline 6 & 3.3608 & 13.9782 & 0.3352 & 10.4876 \\
\hline $\begin{array}{c}\text { Average } \\
\text { Time }\end{array}$ & 5.1250 & 13.9020 & 0.3649 & 10.6567 \\
\hline
\end{tabular}

We can note the average time of the running time in the figure (4.19)the edge detection model is the largest time execution in than the other in the proposed technique .therefore the total time of system running is approximately (19.3919)seconds to detect and following the edges.

From the above, note that the running time of the system is late in approximately ( 5.1250) second because of the preprocessing stage by curvelet via wrapping. The execution time for the edge detection without the preprocessing stage is approximately (13.9020) also the using of the law's texture features is increasing the processing time in large amount approximately (10.6567), also the figure plot(26) shows the values of the rate at the time of execution.

\section{Time in Seconds}

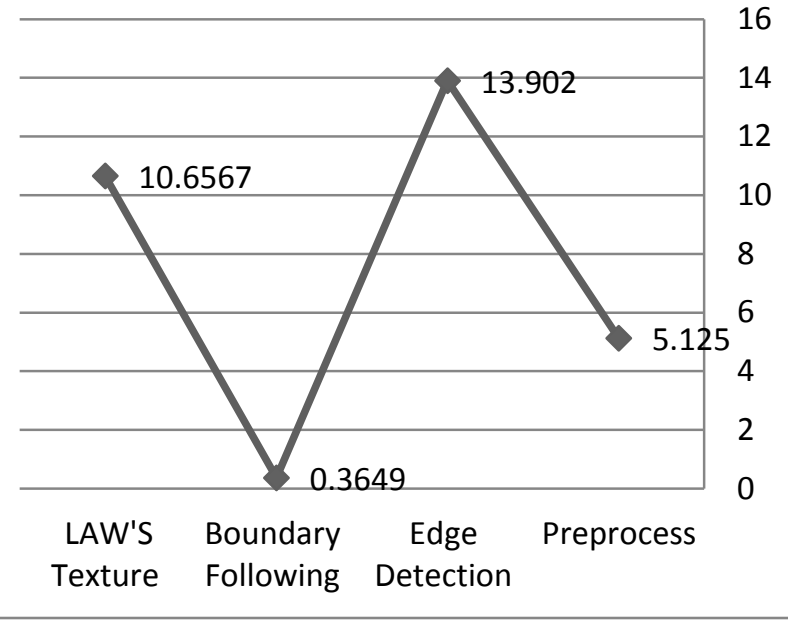

Fig 26: Running Time of Proposed System

\section{CONCLUSIONS}

There are some conclusions that appear from the design of the proposed system, as follows:

1. It gives better results compared to previous models. Also the computing time of this proposed system is very lower compared to the previous methods.

2. Both models proposed in the system rely on the same preprocessing stage, which includes the image preparation stage so this is the proposed system consider an optimal systems for noise elimination before the process of image processing.

3. Boundary can be detected in complex medical images with high accuracy compared to existing traditional models. The extraction of boundary can be useful for doctors in detecting deformities in the human body.

4. The process of analyzing the texture of the image (to find the edge map) and extraction the features is a key step in the system where the analysis of the image texture using a Law's Texture technique and then study the characteristics of the image, there are several techniques used in the analysis of the texture, but the analyzing using Law's Texture method proved efficient in the application which is used in this study but significantly increases processing time.

5. The proposed system has been successfully tested on various types of medical images, including the brain, kidney and heart. It can also be applied to any type of image. However, in noisy and distorted images, the boundary is not defined therefore the process deviation away from the Correct boundary.

6. The advantage of the proposed system in the detection of edges and boundary following is rely on the Average Vector, which eliminates the randomness in the directions calculated from Vector Information previously and make it regular, which will give better results and more accurate and increases the clarity of the edges in the image.

7. Enhancement images using the Curvelet Transform in preprocessing has played a key role in improving the results, making the image better in processing, enhancing the important information in the image and ignoring the nonimportant information which helps in extracting the important 
information and Less complex calculations and faster implementation.

This transform is designed to represent the edges and the elements are treated in multiple directions in each scale. It is more efficient than the traditional transformations. It improves visibility and eliminates unwanted noise to fix contrast and further details in the image, especially the medical image to become more convenient and useful for analysis, and edge detection or features extraction thus improving system performance.

8. The starting point of the boundaries start_point_boundary has an important role in detecting the correct boundaries where there is a direct correlation between them and the validity of the limits detected. Where the lack of quality of some used images lead to wrong detecting to this point and thus to the wrong movement of the boundary.

9. The proposed system can be applied to medical images affected by the disease such as cancer cells in the brain.

\section{REFERENCES}

[1] S. Jose Anand, "An Edge Vector and Edge Map Based Boundary Detection in Medical Images," International Journal of Innovative Research in Computer and Communication Engineering, vol. 1, no. 4, pp. 10501055, June 2013 .

[2] R.Aishwariya, M.Kalaiselvi Geetha, M.Archana, "Computer- Aided Fracture Detection Of X-Ray Images," Journal of Computer Engineering, vol. 15, no. 1, pp. 44-51, 2013.

[3] V.Sai Kumar, V.Vijaya Kishore, "Intensity and Texture Gradient Based Boundary Detection Algorithm for Medical Image," International Journal of Computer Trends and Technology, vol. 6, no. 4, pp. 219-225, Dec 2013 .

[4] Veeralakshmi, Vanitha Sivagami, V.Vimala Devi,R.Udhaya, "Boundary Exposure Using Intensity and Texture Gradient Features," Journal of Computer Engineering, vol. 8, no. 1, pp. 28-33, 2012.

[5] Jamil A. M. Saif, Mahgoub H. Hammad, and Ibrahim A.
A. Alqubati, "Gradient Based Image Edge Detection," International Journal of Engineering and Technology, vol. 8, no. 3, pp. 153-156, June 2016.

[6] Mengmeng Zhang, Qianqian Li, Lei Li and Peirui Bai, "An Improved Algorithm Based on the GVF-Snake for Effective Concavity Edge Detection," Journal of Software Engineering and Applications, vol. 6, no. 4, pp. 174-178, 2013.

[7] Amit D. Purohit, S. T. Khandare, "A SURVEY ON DIFFERENT COLOR IMAGE SEGMENTATION TECHNIQUES USING MULTILEVEL THRESHOLDING," International Journal of Computer Science and Mobile Computing, vol. 6, no. 4, p. 267 273, April 2017.

[8] G.T. Shrivakshan, C. Chandrasekar, "A Comparison of various Edge Detection Techniques used in Image Processing," IJCSI International Journal of Computer Science, vol. 9, no. 5 , No 1, pp. 269-276, September 2012 .

[9] Monica Avlash, Lakhwinder Kaur, "PERFORMANCES ANALYSIS OF DIFFERENT EDGE DETECTION METHODS ON ROAD IMAGES," International Journal of Advanced Research in Engineering and Applied Sciences, vol. 2 , no. 6, pp. 27- 38, June 2013.

[10] Sree Sharmila, Ramar K, "Comparative and Efficient Analysis of Gradient Based Edge Detection Technique in Medical Images," International Journal of Computer Engineering Research, Vols. ISSN:2250-3005, pp. 167170,2012 .

[11] Krit Somkantha, Nipon Theera-Umpon,and Sansanee Auephanwiriyakul, "Boundary Detection in Medical Images Using Edge Following Algorithm Based on Intensity Gradient and Texture Gradient Features," IEEE, 2011.

[12] A.Santhosh Kumar, J.Seetaram, "Medical Images Boundary Detection Using A New Novel Algorithm And Gradient Features," International Journal of Engineering Research \& Technology, vol. 1, no. 8, October - 2012. 